\title{
Empirical Assessments of a tool to support Web usability inspection
}

\author{
Verônica T. Vaz ${ }^{1}$, Tayana Conte ${ }^{2}$, Guilherme H. Travassos ${ }^{1}$ \\ ${ }^{1}$ PESC - COPPE/Universidade Federal do Rio de Janeiro, \\ Rio de Janeiro, Brazil, 21945-970 \\ \{veronica,ght\}@cos.ufrj.br \\ ${ }^{2}$ ICOMP - UFAM, \\ Manaus, Brazil, 69077-000 \\ tayana@icomp.ufam.edu.br
}

\begin{abstract}
Usability is one of the most important software quality attributes regarding its acceptability by end users. It is even more important in the context of Web applications. One way of evaluating application usability is through inspections. The WDP (Web Design Perspectivesbased Usability Inspection Technique) presents evidence of industrial use feasibility; however some computerized support had been suggested by practitioners. Therefore, the WDP Tool was built, aiming to provide automated support the WDP technique application, reducing the effort involved in usability inspections with WDP. This paper presents two observational studies regarding the use of the WDP Tool use, one in vivo and one in vitro, which aimed to analyze the cost-effectiveness of its application and its appropriateness to the industrial environment through the Technology Acceptance Model (TAM). The results provide indications about the feasibility of using the WDP Tool to support usability inspections in real software development projects in the industry.
\end{abstract}

Keywords: Usability Inspections; Software Quality; Web Software Engineering; Experimental Software Engineering.

\section{Introduction}

Usability is one of the software quality attributes. The ISO/IEC 9126 [11] standard defines usability as 'a set of attributes that bear on the effort needed for use, and on the individual assessment of such use, by a stated or implied set of users'. In [7], De la Vara et al. carried out research on the relative importance of each of the software quality attributes. The research results revealed that most subjects considered usability the most important one. In Web applications, usability is even more important because 'Web applications are interactive, user-centred and based on hypermedia, where the user interface plays a central role'[19].

Due to the increasing importance of usability, several evaluation techniques have been proposed to improve its quality [8]. An issue with usability is demand aspect, which can make the system unpleasant, inefficient, onerous, or unable to achieve the users goals in a typical configuration of use [14]. The most commonly adopted methods for the detection of usability problems can be divided into two main categories: (1) Usability Inspections, in which inspectors examine some application aspects in order to identify violations of established usability principles; and (2) Usability Testing, which are evaluation methods that depend on the direct participation of users, such as: Laboratory studies, Think Aloud, and Cooperative Evaluation [2].

In Usability Testing methods the usability problems are discovered by means of observation and interaction with users, while they perform tasks or provide suggestions on the interface design and its usability. In Usability Inspections, problems are discovered by usability experts guided by inspection techniques [2]. Usability inspections cost less than usability testing because they only need practitioners with good usability knowledge to be performed, instead of special equipment or a laboratory [25]. Different usability inspection techniques have been proposed in the technical literature, such as: Heuristic Evaluation [18], Cognitive Walkthrough [21], and Guidelines and Checklists [6]. Amongst those, Heuristic Evaluation is one of the most popular [18]. In Heuristic Evaluation, 
inspectors analyze system conformity by considering a set of heuristics representing quality patterns defined by usability experts.

The WDP (Web Design Perspective-based Usability Evaluation) is an usability inspection technique based on heuristics [2]. The WDP technique was developed through an empirical approach [24] to reduce the risk of transferring an immature software technology from the Academy to the industry [4]. Empirical studies on the WDP technique also allowed the identification of the main difficulties inspectors faced when using the technique, such as: (1) the effort needed for a detailed description of detected usability defects; and (2) the fact that it was necessary to change the focus of attention, going from the evaluated application to the written description of WDP technique, and the evaluation report. Such issues motivated the development of tools to support the application of the WDP technique. Some tools have been proposed with this goal, such as the APIU [22], Interactive WE [9] and the WDP technique content consult assistant [10]. However, we considered that the issues previously identified had not yet been fully addressed by these tools, and proposed the WDP Tool. The WDP Tool aims to support the WDP inspection process, to reduce the effort needed for its application, therefore enhancing its adherence to an industrial environment.

This paper presents two observational studies using the WDP technique within the WDP Tool. The first study was conducted in vitro, with students from a software engineering MSc course at the Federal University of Rio de Janeiro. The second one was conducted in vivo, in a Brazilian brokerage firm. Both studies aim to characterize the efficiency of the WDP Tool and evaluate its feasibility when used in real software development projects. Moreover, this paper also describes how the WDP Tool has been evaluated in terms of its adequacy to an industrial environment through the TAM (Technology Acceptance Model) model [5]. Studies indicate the feasibility of successfully using the WDP technique along with the WDP Tool to support usability inspections in real software projects.

The remainder of this paper is organized as follows. Section 2 presents the background on usability inspections using the WDP technique, and the motivation for this research. Section 3 describes the technologies we used in the development of the WDP tool, and Section 4 presents the WDP tool and its main functionalities. In Sections 5 and 6 , we describe the empirical studies evaluating the WDP tool. Moreover, Section 7 presents the results analysis regarding the perceptions of usefulness and ease of use of the WDP tool according to the TAM model [5]. Then, Section 8 provides a brief comparison with related tools. Finally, we give our conclusions and future work paths in Section 9.

\section{Usability Inspection with the WDP Technique}

\subsection{The WDP Technique}

According to Zhang et al. [27] it is hard for an inspector to identify all the different usability problems at the same time. In order to address this issue, Zhang et al. [27] proposed an usability inspection technique based on perspectives called Use-Based Reading (UBR), in which each inspection session focuses on a subset of usability aspects covered within a usability perspective. The hypothesis behind the use of perspectives is that, due to the focus of each perspective, each inspection session will have an increased percentage of identified usability problems related to the used perspective. Furthermore, the combination of different perspectives could assist in identifying more usability problems than the same amount of inspections sessions using a generic inspection technique [25].

Combining such hypotheses with the set of usability heuristics proposed by Nielsen [18], Conte et al. [2] developed the WDP technique. Empirical results presented in [2] show that the WDP technique is significantly more effective than, and as efficient as, Nielsen's Heuristic Evaluation for the inspection of Web applications. The WDP technique uses perspectives (presentation, concept, and navigation) that are proper for Web projects, and that can be used as a guide to interpret the heuristics with a specific focus on Web applications (Table 1).

The combination of Heuristics $(\mathrm{H})$ and Perspectives $(\mathrm{P})$ allows the WDP to define HxP pairs (Table 2), in which each heuristic is analyzed under the related perspective specific focus. It is worth noting that not all associations between heuristics and perspectives make sense; therefore, in that case, there are empty cells in the table.

For each HxP pair, the WDP technique defines a set of features to be evaluated. Fig. 1 shows part of the WDP technique, in which readers can view the focus difference regarding Heuristic 9 ("Help users recognize, diagnose and recover from errors") according to each of the perspectives, by pairs P.9, C.9, and N.9, respectively. 
Table 1: Web Design Perspectives related to Usability Evaluation [3]

\begin{tabular}{ll}
\hline Web Design Perspectives & Usability Focus \\
\hline \multirow{3}{*}{ Conceptual } & $\begin{array}{l}\text { Relates to the clarity and the conciseness of the elements in the problem } \\
\text { domain. From this perspective, the usability is satisfactory if different users } \\
\text { easily grasp domain terms, preventing mistakes caused by ambiguous, } \\
\text { inconsistent or unknown terms. }\end{array}$ \\
& $\begin{array}{l}\text { Relates to how consistently the information is presented to the user. From this } \\
\text { perspective, the usability is satisfactory if the arrangement of the interface's } \\
\text { elements allows users to accomplish their tasks effectively, efficiently, and } \\
\text { presentation }\end{array}$ \\
& $\begin{array}{l}\text { Relasantly. } \\
\text { perspective, the usability is satisfactory if the navigation options allow users } \\
\text { to accomplish their tasks effectively, efficiently, and pleasantly. }\end{array}$ \\
\hline
\end{tabular}

Table 2: Heuristic x Perspective (HxP) pairs

\begin{tabular}{|c|c|c|c|}
\hline \multirow{2}{*}{ Heuristic } & \multicolumn{3}{|c|}{ Related to the Perspectives: } \\
\hline & Presentation & Concept & Navigation \\
\hline Visibility of system status & P.1 & C.1 & \\
\hline Matching between system and real world & P.2 & C. 2 & \\
\hline User control and freedom & & & N.3 \\
\hline Consistency and standards & P.4 & C. 4 & \\
\hline Error prevention & P.5 & & N.5 \\
\hline Recognition rather than recall & P.6 & & \\
\hline Flexibility and efficiency of use & P.7 & & N.7 \\
\hline Aesthetic and minimalist project & P.8 & & \\
\hline Help users recognize, diagnose and recover from errors & P.9 & C.9 & N.9 \\
\hline Help and documentation & P.10 & C. 10 & N.10 \\
\hline
\end{tabular}

\section{P.9. Help users recognize, diagnose, and recover from errors}

- Evaluate if the error messages are presented in a consistent way according to adopted standards.

- Evaluate if the user's attention is directed to the error messages.

- Evaluate if the alternative solutions to an error are clear to the user.

C.9. Help users recognize, diagnose, and recover from errors

- Evaluate if error messages provide recovery steps or procedures easily understood by users.

- Evaluate if error messages are simple, and use terms, symbols, words or sentences of problem domain.

N.9. Help users recognize, diagnose, and recover from errors

- Evaluate if the system shows how to access the alternative solutions presented on error messages.

Figure 1: Part of the WDP technique (adapted from [4])

\subsection{The Inspection Process using the WDP Technique}

The inspection process using the WDP technique is divided into five main activities based in [23]. Table 3 describes each one of them. 
Table 3: Activities of the inspection process of the WDP technique

\begin{tabular}{ll}
\hline Activity & Description \\
\hline Planning & $\begin{array}{l}\text { Defines the inspection scope (choosing main interactions and modules in the } \\
\text { application), and preparing the material for inspection. Furthermore, to select } \\
\text { the inspectors, train them in the WDP technique and assign the tasks. }\end{array}$ \\
Detection & $\begin{array}{l}\text { Each Inspector carries out this activity individually. The inspector, guided by } \\
\text { the HxP pairs defined in WDP technique should look for usability problems in } \\
\text { the selected interactions. The problems identified should be reported as } \\
\text { discrepancies in the inspection report. }\end{array}$ \\
Collection & $\begin{array}{l}\text { Elimination of repeated discrepancies (discrepancies found by more than one } \\
\text { inspector). Creating a list containing only unique discrepancies (without } \\
\text { duplicated ones). } \\
\text { The inspectors and the Web application responsible review the reported } \\
\text { discrepancies, verifying whether they are real usability defects or false } \\
\text { positives. } \\
\text { Analysis of the degree of severity in the defects and deciding their correction } \\
\text { priority. }\end{array}$ \\
\hline
\end{tabular}

\subsection{The Detection Activity Using the WDP Technique}

In the context of this work, the detection activity was studied with special attention, as previous studies with WDP [2][4][25] indicated that this activity was the most effort-intensive activity in the inspection process. In order to perform the detection of discrepancies, the inspector should use the following supporting material:

- The WDP technique description, which completely describes the technique (the complete technique description can be found in [2]). Usually, inexperienced inspectors tend to use this description to guide them through the detection activity. Nevertheless, experienced inspectors use it as a support instrument for the classification of defects.

- The inspection script, which identifies, in terms of activities and steps, which Web application functionalities should be inspected. The inspection script is prepared according to the inspection plan.

- The discrepancies report, which represents the detection activity product. It contains all the discrepancies found by the inspector in the inspected Web application. For each discrepancy, the inspector should inform: the activity from the inspection script that was being performed when he/she found the discrepancy, the step of the activity, the discrepancy classification (the HxP pair), the problem description, its repetition (if the activity gets repeated in other activities, the inspector should report which one of them), and the degree of severity (using the severity scale proposed by Nielsen [18]). Furthermore, the report should complement the information by informing the inspector ID and total inspection time, which should be measured by the inspector him/herself. Fig. 2 shows a report example.

\begin{tabular}{|c|c|c|c|c|c|c|}
\hline & & & \multicolumn{2}{|c|}{ Discrepancies Report } & & \\
\hline Inspector: & John Doe & & & & & \\
\hline Time Spent: & 102 & $\min$ & & & & \\
\hline Number & Activity & Step & Pair HxP & Description of the Encountered Problem & Is the problem repeated? & Severity \\
\hline 1 & AT02 & 4 & C2 & $\begin{array}{l}\text { There is no Definition of the various } \\
\text { acronyms. For instance, DIEF, INSS and } \\
\text { others are not defined. }\end{array}$ & ter & Tenuous \\
\hline 2 & AT04 & 2 & C9 & $\begin{array}{l}\text { When values larger than } 100.00 \text { are } \\
\text { informed, the system shows an error } \\
\text { message: ("The current value is invalid") } \\
\text { that does not indicate that the problem } \\
\text { is that the value is larger than } 100.00 \text {. }\end{array}$ & No & Severe \\
\hline
\end{tabular}

Figure 2: Example of an inspection report 
In general, the work of the inspector concerns: (1) consulting the technique and the inspection script using text editors; (2) inspecting the Web applications using a Web browser; and (3) pointing out the identified problems in the discrepancies report. Furthermore, the inspector should also measure the time spent in the detection activity.

Based on the observations in the WDP technique empirical studies [2][4][25], we concluded that this constant context change (from the Web browser to the text editor and the report spread sheet) is one of the factors that make the detection activity tiring and subject to errors. Furthermore, the inspectors usually complain about the demanded effort to thoroughly describe each of the usability problems found. These difficulties represent our main motivation for developing the WDP Tool. We will describe the way in which the tool deals with each of these issues in Section 4.

\section{Technology Bases}

The following technologies were used as bases for the development of the WDP Tool:

Firefox: The WDP Tool aims to specifically support the inspection of Web applications. Therefore, it is interesting to integrate its tool support to a technology capable of accessing the Web. This feature aims to reduce the need for context changing in the detection activity. As a result, the WDP Tool has been developed as an extension of the Web browser. The most commonly used browsers are: Microsoft Internet Explorer (IE), Mozilla Firefox, and Google Chrome[29]. Despite IE being the browser with the biggest market share, it does not offer enough resources to support developers willing to extend its functionalities. Consequently, we chose Firefox as the development basis for the WDP tool due to the support it provides for the development of its extensions, and as it is the secondly mostly used browser [15].

ISPIS (Infra-estrutura de Suporte ao Processo de Inspeção de Software, or Software Inspection Process Support Infrastructure): A framework to support software inspection software that can be used by distributed teams to inspect different artefacts throughout the software development life cycle [12]. The ISPIS framework provides support to all activities in the inspection process, from planning to rework and follow-up of results. However, as a generic inspection framework, it does not deal with the particularities of any specific inspection technique (such as WDP). In order to tackle this limitation, the ISPIS provides an integration mechanism, allowing discrepancies that were identified using other tools (such as the WDP tool) to be integrated into the ISPIS framework (see Fig. 3). In that sense, we found that it would be valuable to integrate the WDP Tool with the ISPIS. This integration is done through the generation of a XMI file, with all the discrepancies identified by the inspector in the detection phase (using the WDP Tool), which can be automatically loaded and used within the ISPIS framework.

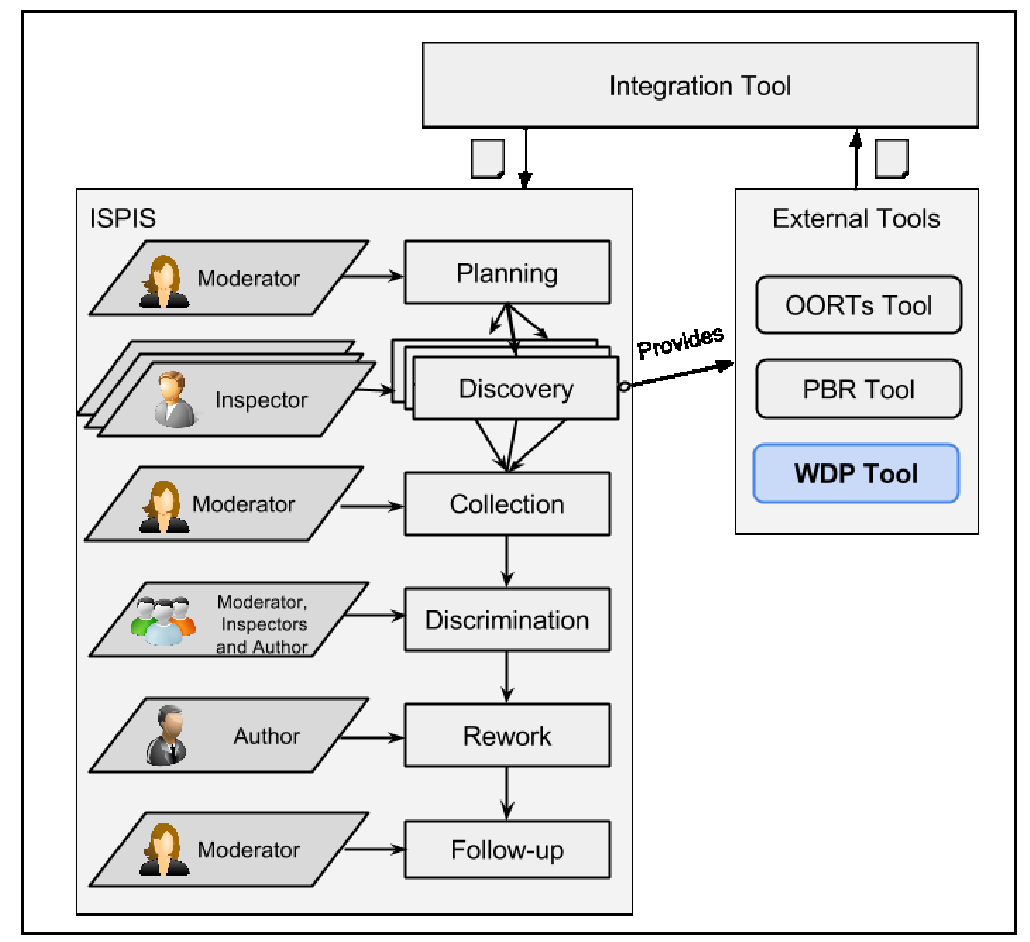

Figure 3: ISPIS structure and integration schema (adapted from [12]) 
The WDP technique content consult assistant [10] provides access to the WDP technique description, showing its content in small parts and allowing the navigation through links, which make the reading process less tiring. The inspector may choose which perspective he/she wants to see, and pairs for this perspective are presented in numerical order. Usually $\mathrm{HxP}$ pairs are evaluated in this order: first the Presentation perspective pairs, as presentation is the most immediate observable characteristic of Web application interfaces; secondly, the inspector evaluates if he/she can understand what is being presented, following the Conceptual perspective HxP pairs; and finally, the inspector navigates to other pages, evaluating the Navigation perspective pairs. Although this is the suggested order, the inspector is free to follow his/her own order.

For each HxP pair, the assistant shows the points that should be evaluated, links to examples and navigation buttons (see Fig. 4). This window is shown in the screen right bottom, in order to minimize its interference with the Web applications that are being inspected (see Fig. 5).

\section{P.6) Recognition rather than recall}

1) Evaluate if system interface allows the user to identify key information during a task.

2) Evaluate if it is easy to recognize/identify data already provided.

3) Evaluate if it is easy to recognize/identify the correct option to reach a goal.

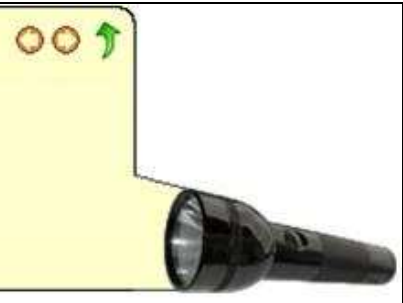

Figure 4: Content consult assistant for the WDP technique over the Firefox browser window

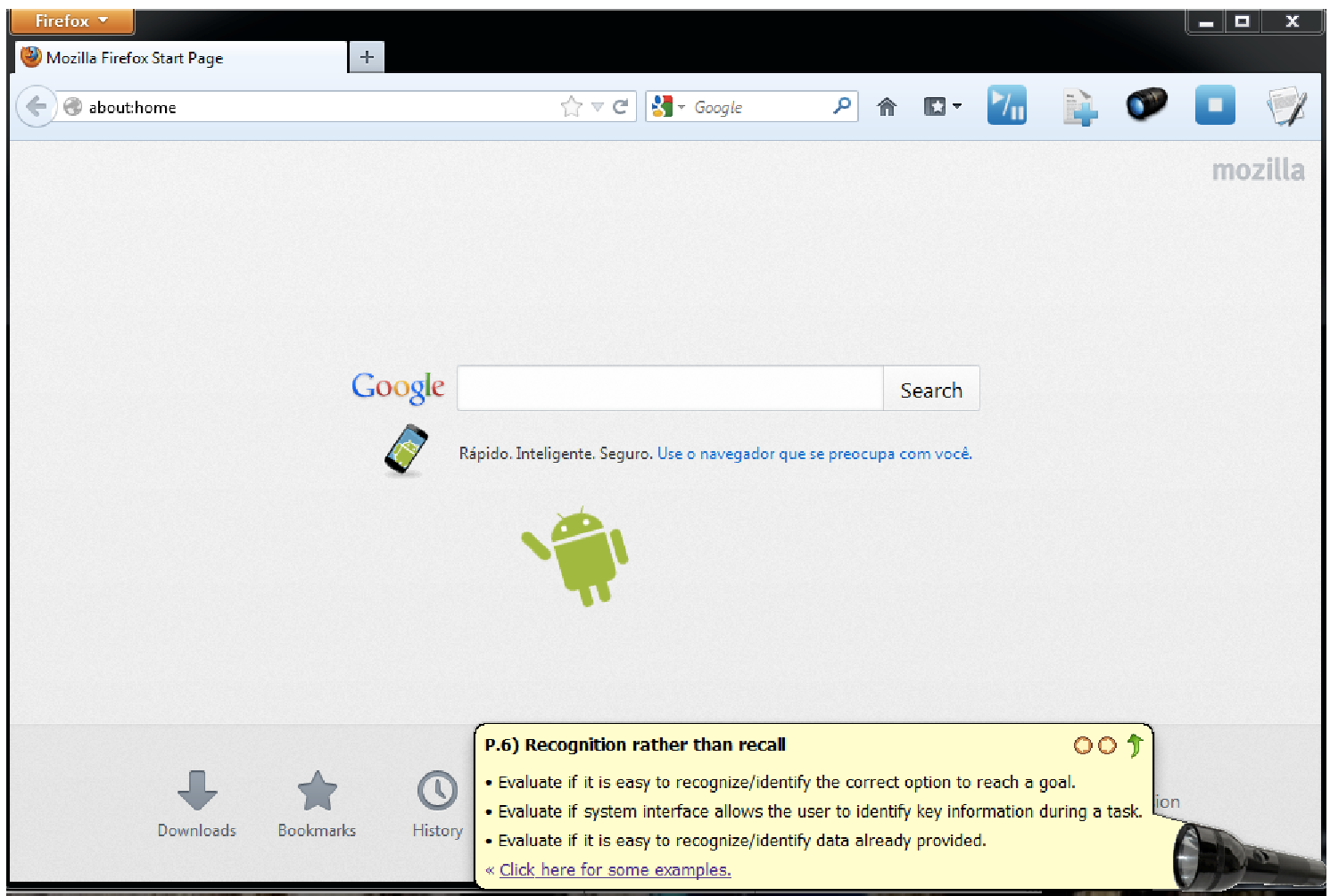

Figure 5: Content consult assistant for the WDP technique on a Firefox browser window

The development of this assistant was the initial stage of this work in providing automated support in the application of the WDP technique. The WDP tool is an evolution of this work, and therefore, was developed as an integration to the assistant.

\section{The WDP Tool}

The requirements for the development of the WDP tool were directly derived from each of the problems identified in the application of the WDP technique (see Table 4). 
Based on these requirements, we proposed a set of functionalities to be offered by the tool (see Table 5). Fig. 6 shows the location of such functionalities in the tool's menu on the browser's tool bar. The only feature we do not show in this menu is the "Edit tool preferences" functionality, which is shown in another browser tool bar.

Table 4: Origin of WDP Tool requirements

\begin{tabular}{l|l}
\hline Problems of Usability Inspections & Derived Requirements \\
\hline $\begin{array}{l}\text { Effort needed in the detection activity: the inspector } \\
\text { should leave the context of the evaluated application to } \\
\text { report the usability problem in a spread sheet editor. }\end{array}$ & $\begin{array}{l}\text { discrepancies in the Web browser window. } \\
\begin{array}{l}\text { Effort needed in thoroughly describing the reported } \\
\text { discrepancy during the detection stage. }\end{array}\end{array} \begin{array}{l}\text { The tool should allow taking screenshots of the } \\
\text { system's screen, allowing inspectors to indicate } \\
\text { usability defects. This feature will reduce the } \\
\text { inspector's effort in describing the identified problem. }\end{array}$ \\
\hline $\begin{array}{l}\text { Effort needed in reproducing the error in the system } \\
\text { during the discrimination meeting. }\end{array}$ & $\begin{array}{l}\text { Each reported discrepancy should be associated to a } \\
\text { screenshot, reducing the need for accessing the system } \\
\text { in order to review the identified problem. }\end{array}$ \\
\hline $\begin{array}{l}\text { Need for leaving the evaluated application context in } \\
\text { order to consult the technique contents. }\end{array}$ & $\begin{array}{l}\text { Integration with the tool developed by [10], which } \\
\text { allows the visualization of the content of the technique } \\
\text { in a more comfortable way, without closing the Web } \\
\text { browser. }\end{array}$ \\
\hline Need for measuring the inspection time. & \begin{tabular}{l} 
Automatically measuring the inspection time. \\
\hline
\end{tabular}
\end{tabular}

Table 5: Functionalities of WDP Tool

\begin{tabular}{ll}
\hline Functionality & Description \\
\hline Edit tool preferences & $\begin{array}{l}\text { Allows the inspector to configure some tool parameters: format of the } \\
\text { discrepancies report (CSV or XMI - for the integration with the ISPIS[12]), } \\
\text { directory where the report will be saved, and program for image editing (for } \\
\text { screenshots edition, such as MS Paint [20] or another)) }\end{array}$ \\
\hline Start inspection & $\begin{array}{l}\text { Starts measuring the inspection time, and registering the inspector and the } \\
\text { evaluated application's name. }\end{array}$ \\
\hline Report Discrepancy & $\begin{array}{l}\text { Allows the user to report an encountered usability problem during the } \\
\text { inspection. Allows him/her to automatically save a system screenshot in which } \\
\text { the problem was found, and edit such image by using an external image editor } \\
\text { (which will be configured using the tool's preferences). }\end{array}$ \\
\hline Edit discrepancies report & $\begin{array}{l}\text { Allows the discrepancies report to be opened in a text editor for consultation } \\
\text { or edition. }\end{array}$ \\
\hline Consult the WDP technique \\
contents
\end{tabular}




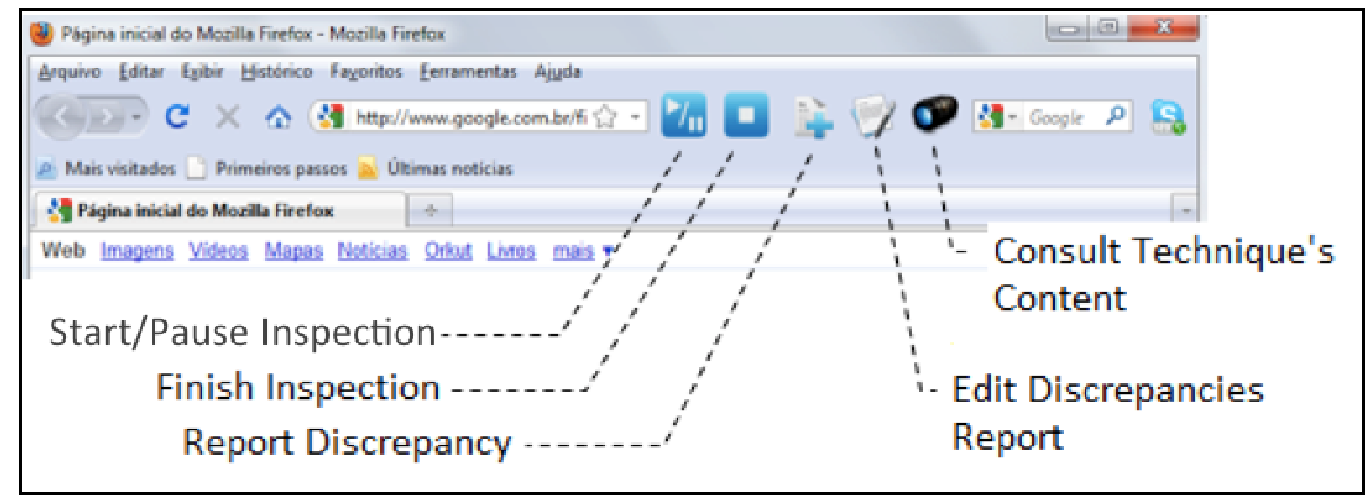

Figure 6: Buttons of the WDP tool in the browser

The inspection starts when the user clicks the "Start Inspection" option. Then, the WDP Tool asks the inspector to enter his/her name and the name of the application being inspected. Afterwards, the tool starts counting the inspection time and creates a new directory in which it will store the discrepancies report. Later, the inspector carries out the detection activity, following the steps defined in the inspection script and verifying each of the suggested HxP pairs of the technique. Furthermore, if the inspector wants it, he/she can view the technique by clicking on the "Consult the Technique Contents" option, which will open the technique consult assistant. In this stage, the assistant remains open throughout the inspection, without interfering with the WDP tool or the inspection. So far the assistant provides support only for the technique's content, and not to the inspection script. However, as the assistant is configured through an XML, it is possible to also include the script steps in its content.

When finding a usability problem, the inspector should point it using the "Report Discrepancy" option. The system will show a form that can be used to describe and classify the discrepancy, as shown in Fig. 7. It is worth pointing that, after describing the encountered problem, the user can either: (1) not save, (2) save, and (3) save and edit an image of the window in which the discrepancy was found. Then, the system will automatically take a screenshot and save it in the inspection directory. If the user selects the "Save and Edit" option, the system will also open the file containing the screenshot with an external image editor. In this case, the inspector can make drawings and marks that can indicate and facilitate the understanding of the discrepancy (see Fig. 8).

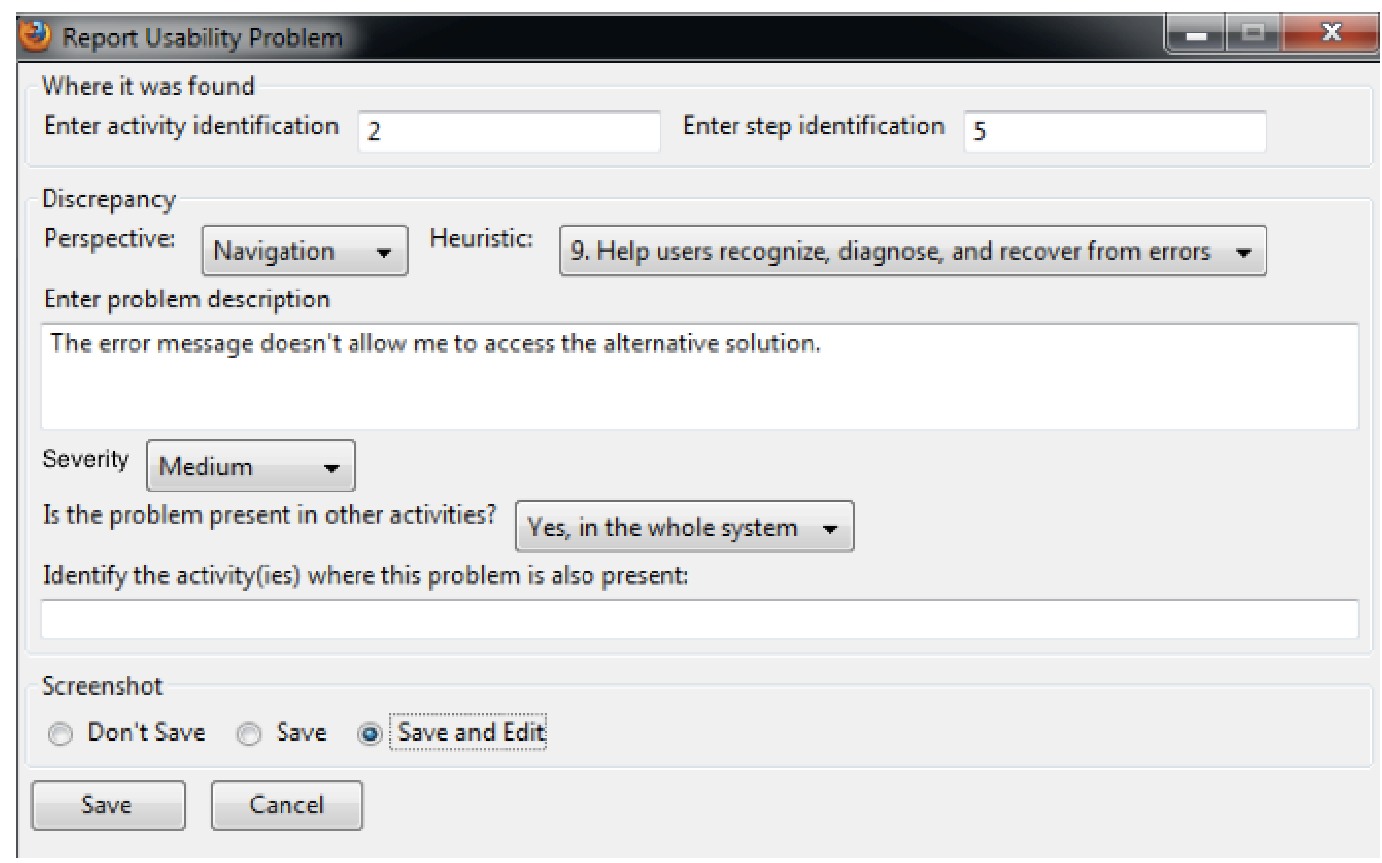

Figure 7: Window within the WDP tool that can be used to report a discrepancy 


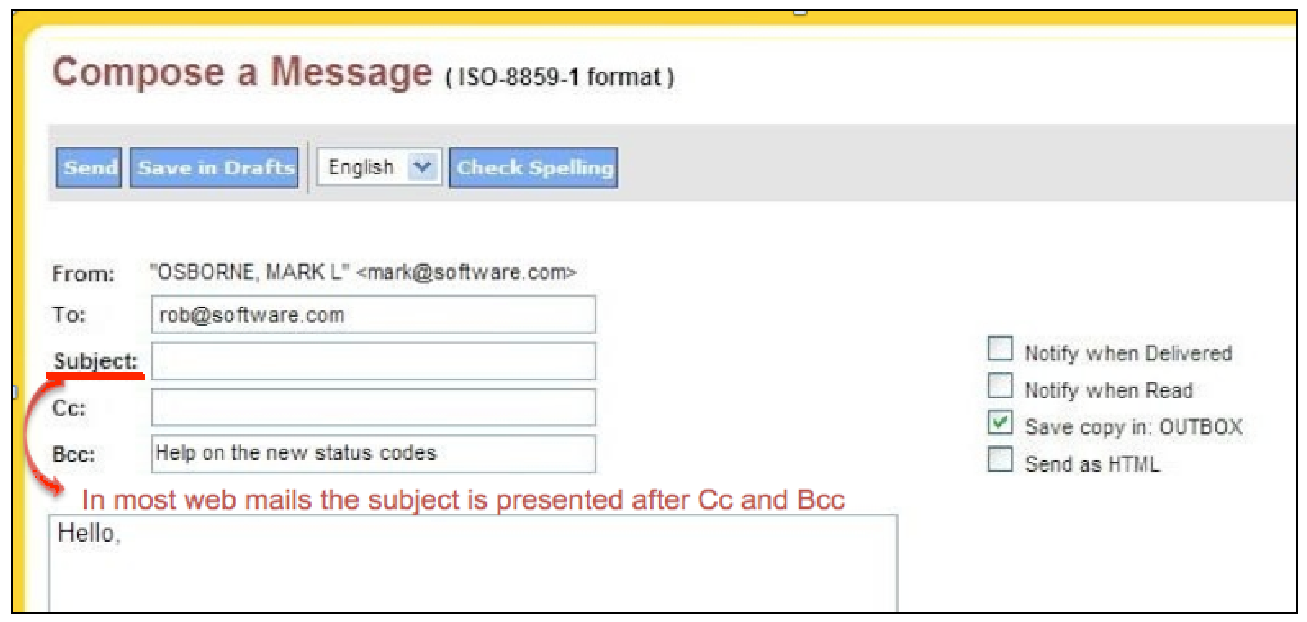

Figure 8: Example of a screenshot that has been edited to facilitate the understanding of the reported discrepancy

After finishing the inspection, the inspector should click the "Finish Inspection" option. The report containing all the reported discrepancies will be available in the inspection directory. The inspector can open the report directly from its folder, or use the "Edit Discrepancies Report" option in the WDP Tool. Fig. 9 shows an example of a report created by the tool in the CSV format. The images saved for each discrepancy are shown in the report through links that point to the related image file.

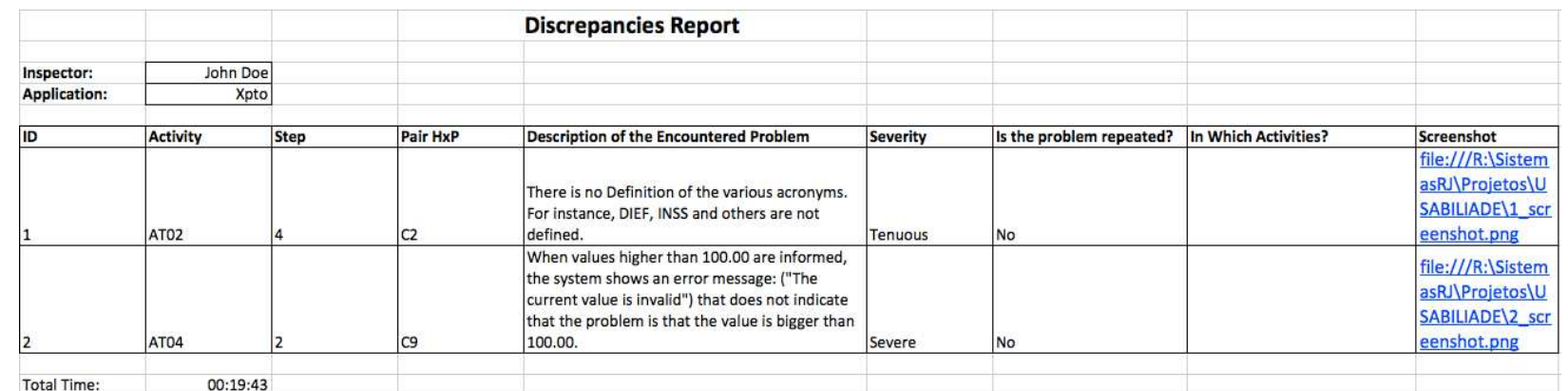

Figure 9: Report that has been generated using WDP Tool

The WDP Tool is available for download at [28] under the Mozilla Public License Version 1.1[16] license for those interested in its use.

\section{First Observational Study}

We carried out two empirical studies to evaluate the WDP Tool. In these studies we aimed to observe the feasibility of the WDP Tool application to support usability inspections, using the WDP technique. We carried out the first empirical study in vitro, with eight Software Engineering MSc students who carried out an inspection in the MPS.Br [17] website. None of the subjects had previous experience with usability inspections. All subjects received the same training and the same inspection script. Each subject inspected the system individually, using the WDP technique along with the WDP Tool. The goals of this first study are described using the GQM (Goal Question Metric) paradigm [1] shown in Table 6. 
Table 6: Goals of the first empirical study (GQM based)

\begin{tabular}{|c|c|}
\hline Analyze & The application of the WDP technique with the WDP Tool \\
\hline With the purpose of & Characterizing \\
\hline In terms of & $\begin{array}{l}\text { Efficiency (number of defects divided by the time spent to find them) in the } \\
\text { detection stage. }\end{array}$ \\
\hline From the point of view of & Software Engineering Researchers \\
\hline In the context of & $\begin{array}{l}\text { MSc students in Software Engineering evaluating the usability of a real Web } \\
\text { application used to provide information on the MPS.Br program to the general } \\
\text { public. }\end{array}$ \\
\hline Analyze & The application of the WDP technique with the WDP Tool \\
\hline With the purpose of & Understanding \\
\hline In terms of & Perception of usefulness and ease of use of the tool \\
\hline From the point of view of & Usability inspectors \\
\hline In the context of & $\begin{array}{l}\text { MSc students in Software Engineering evaluating the usability of a real Web } \\
\text { application used to provide information on the MPS.Br program to the general } \\
\text { public }\end{array}$ \\
\hline
\end{tabular}

Following the first goal of the study, we collected data regarding the time each inspector spent to carry out the inspection, and the amount of discrepancies and real defects found per inspector. The number of real defects is the number of discrepancies pointed by the inspector minus the number of false-positives (discrepancies that are not real usability problems). The false-positives are identified during the inspection discrimination stage (see Table 3 ). We have summarized the collected data in Table 7.

Table 7: Quantitative results from the first empirical study

\begin{tabular}{|lllll}
\hline & Effort(hh:mm:ss) & \multicolumn{1}{c}{$\begin{array}{c}\text { No. of } \\
\text { Discrepancies }\end{array}$} & $\begin{array}{c}\text { No. of Real } \\
\text { Defects }\end{array}$ & $\begin{array}{c}\text { Efficiency } \\
\text { (defects/hour) }\end{array}$ \\
\hline Inspector 1 & $00: 54: 41$ & 13 & 8 & 8,73 \\
\hline Inspector 2 & $00: 52: 37$ & 20 & 17 & 19,25 \\
\hline Inspector 3 & $00: 42: 03$ & 9 & 7 & 10,00 \\
\hline Inspector 4 & $00: 59: 37$ & 19 & 11 & 11,00 \\
\hline Inspector 5 & $01: 01: 26$ & 13 & 12 & 11,80 \\
\hline Inspector 6 & $00: 44: 47$ & 5 & 5 & 6,67 \\
\hline Inspector 7 & $01: 01: 10$ & 16 & 13 & 12,79 \\
\hline Inspector 8 & $01: 32: 53$ & 14 & 12 & 7,74 \\
\hline Average & $\mathbf{0 0 : 5 8 : 3 9}$ & $\mathbf{1 3 , 6 2 5}$ & $\mathbf{1 0 , 6 2 5}$ & $\mathbf{1 1 , 0 0}$ \\
\hline
\end{tabular}

When analyzing the data, it is possible to see that the average efficiency of WDP Tool was of about 11 defects per hour of inspection. It would be interesting to compare such efficiency with the average efficiency reported in other studies on the WDP technique. However, such comparison is hard due to the fact that the efficiency indicator directly depends on the inspected application, as some applications can have more defects than others. Therefore, such comparison will only be possible after obtaining data from other studies involving the same application. We propose those studies as future work in Section 9.

Fig. 10 shows the qualitative results from this study. We collected the qualitative data in this study through the subjects' answers to a follow-up questionnaire to evaluate two aspects: (a) perceived usefulness: the degree in which a person believes that the technology could improve his/her performance at work, and (b) perceived ease of use: the degree in which a person believes that using a specific technology would be effortless [13]. Seven out of eight subjects answered the questionnaire. For each questionnaire we used a four level Likert scale. We did not use a five level Likert scale with an intermediate level, as suggested by [13], as this neutral level does not provide information 
regarding the side to which the subjects is inclined (either positive or negative). We can see that the subjects had positive answers regarding the usefulness and ease of use of the tool. Furthermore, all subjects stated that they would use the tool again if they were to perform another usability inspection using the WDP technique.

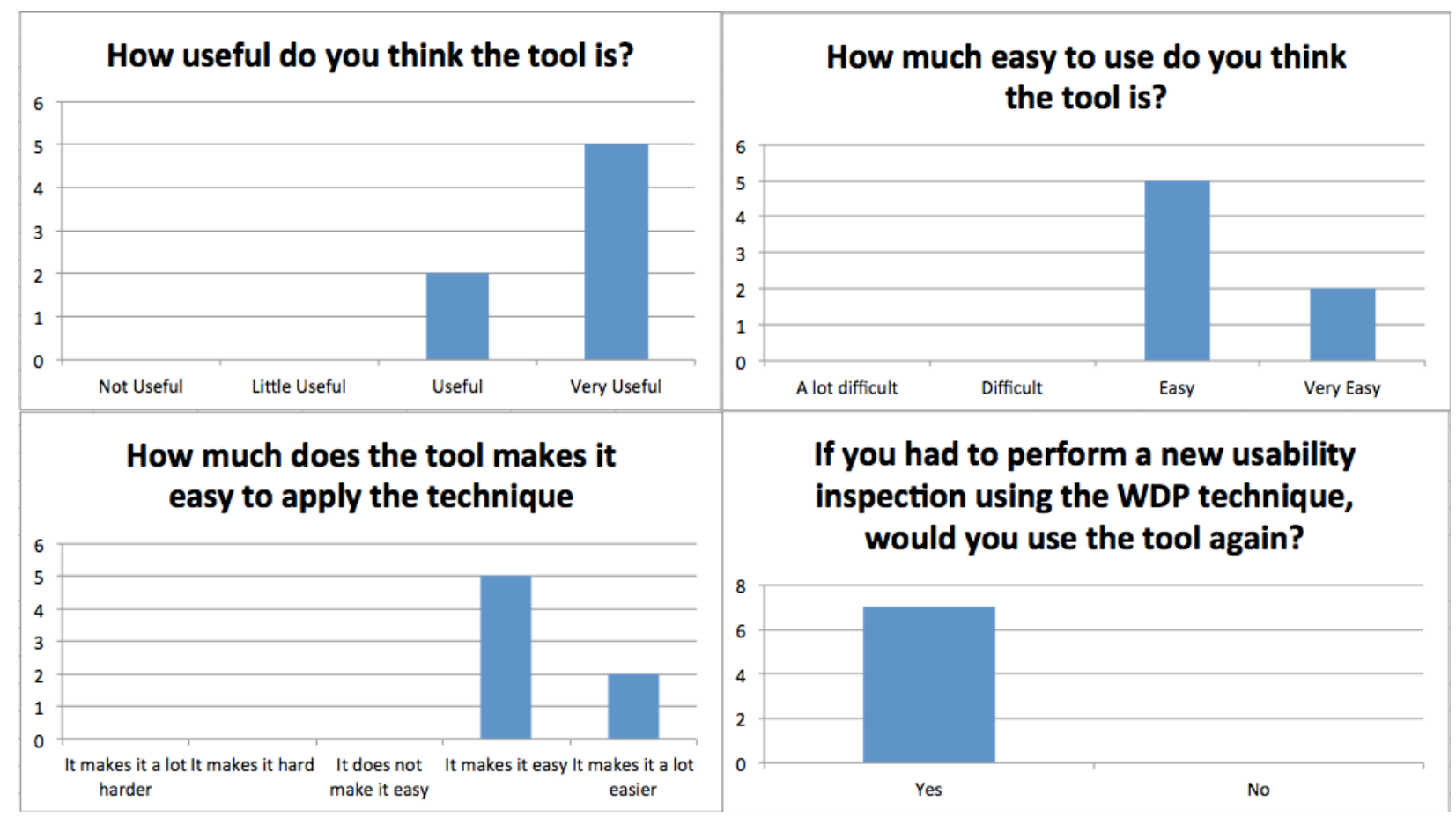

Figure 10: Qualitative results from the first empirical study

\section{Second Observational Study}

Having characterized the use of the WDP Tool in the first empirical study, we carried out the second one, in vivo, in a firm from the financial market area (a brokerage firm) in Rio de Janeiro, Brazil. In this company, there are about 40 collaborators, 10 of them being software developers. Moreover, the company develops software applications related to the financial market for its own use. We chose two of the developed applications as the software to be inspected in this study. The selection criterion was the opinion of an internal project manager of the company, who considered these applications to be critical and needed to improve their usability to reduce user errors and improve user satisfaction. Five of the company developers were selected as inspectors. These inspectors were divided into two teams, each of them allocated to evaluating one of the selected applications (we refer to the applications as System 1 and System 2). There were a total three inspectors in each team and one of the developers was a member of both teams. We carefully divided the subjects of this study so that none of the developers would inspect a functionality he/she had developed. The goals of this study are shown in Table 8.

Table 8: Goals from the second empirical study

\begin{tabular}{|l|l|}
\hline Analyze & The application of the WDP technique with the support of the WDP tool \\
\hline With the purpose of & Understanding \\
\hline In terms of & Perception of usefulness and ease of use of the tool \\
\hline From the point of view of & Inspectors (developers from the organization) \\
\hline In the context of & $\begin{array}{l}\text { Usability evaluation of two real Web applications for the financial market that } \\
\text { have been developed by the organization }\end{array}$ \\
\hline
\end{tabular}

Table 9 presents subject characterization and Table 10 shows the quantitative results from this study. It is worth noting that one of the subjects did not carry out the inspection in System 2. This inspector was allocated to another task during the study execution and was not able to participate in the inspection. Furthermore, System 2 presented 
some functional defects that were not related to usability, but that were identified during the inspection. Therefore, we have separated the identified defects in usability problems and other problems (see Table 10). The overall inspection allowed the identification of 33 unique defects in System 1, and 31 unique defects in System 2 (please note that the sum of defects per inspector is greater than the total number of unique defects in System 2, as some defects where pointed out by more than one inspector). The company used these results to improve the quality of its applications.

Table 9: Inspector Characterization

\begin{tabular}{lccccc}
\hline & $\begin{array}{c}\text { Previous } \\
\text { usability } \\
\text { knowledge } \\
(\mathbf{1 - 5} \text { scale) }\end{array}$ & $\begin{array}{c}\text { Experience with } \\
\text { software } \\
\text { inspections } \\
(\mathbf{1 - 4} \text { scale) }\end{array}$ & $\begin{array}{c}\text { Experience with } \\
\text { software } \\
\text { development (years) }\end{array}$ & $\begin{array}{c}\text { Time } \\
\text { working in } \\
\text { this } \\
\text { company }\end{array}$ & $\begin{array}{c}\text { Previous knowledge } \\
\text { of Mozilla Firefox } \\
\text { Web Browser } \\
(\mathbf{1 - 3} \text { scale) }\end{array}$ \\
\hline Inspector 1 & 4 & 3 & 3 & 1 month & 3 \\
\hline Inspector 2 & 2 & 1 & 2.5 & 6 months & 3 \\
\hline Inspector 3 & 2 & 1 & 4 & 1 year & 3 \\
\hline Inspector 4 & 2 & 1 & 1 & 4 months & 3 \\
\hline
\end{tabular}

Table 10: Quantitative results from the second empirical study

\begin{tabular}{llll}
\hline System 1 & No. of Discrepancies & No. of Usability Defects & No. of Other Defects \\
\hline Inspector 1 & 31 & 25 & 0 \\
\hline Inspector 2 & 5 & 4 & 0 \\
\hline Inspector 3 & 9 & 4 & 0 \\
\hline System2 & No. of Discrepancies & No. of Usability Defects & No. of Other Defects \\
\hline Inspector 1 & 25 & 16 & 4 \\
\hline Inspector 4 & 18 & 12 & 4 \\
\hline
\end{tabular}

As expected, more experienced inspectors were able to detect more defects. However, all inspectors agreed on the tool's usefulness to support the inspection regardless of their experience level, as it will be shown in the next section. We consider the results obtained useful and as indicators of the feasibility of the WDP Tool.

The next section presents the qualitative results from this empirical study. As we wanted to describe in detail the application of the Technology Acceptance Model we present this part of the study in a separate section.

\section{Applying the TAM (Technology Acceptance Model)}

The Technology Acceptance Model (TAM), as proposed by Davis [5], has been applied in the evaluation of several new technologies [26]. We used this model as a basis for the qualitative analysis in this second study, aiming to evaluate the indicators of perceived usefulness and ease of use. The reason for focusing on these indicators is that, according to Davis [5], these aspects are strongly correlated to user acceptance.

In this second observational study we prepared a post-inspection questionnaire, which was based in the questionnaires applied by Conte et al. [4]. In this questionnaire, the subjects had to answer how much they agreed with each of the statements regarding the tool's usefulness: (i) the tool improves the productivity of the inspection; (ii) the tool makes facilitates the application of the technique; (iii) the tool makes it easier to understand the reported discrepancies. They also indicated their agreement with the statements regarding the tool's ease of use: (i) the tool was easy to use. In order to assess the degree of agreement, the inspectors selected one of the following values from a Likert scale: (1) totally disagree, (2) greatly disagree, (3) partially disagree, (4) partially agree, (5) greatly agree, and (6) totally agree. Fig. 11 shows the overall results from the questionnaire. 


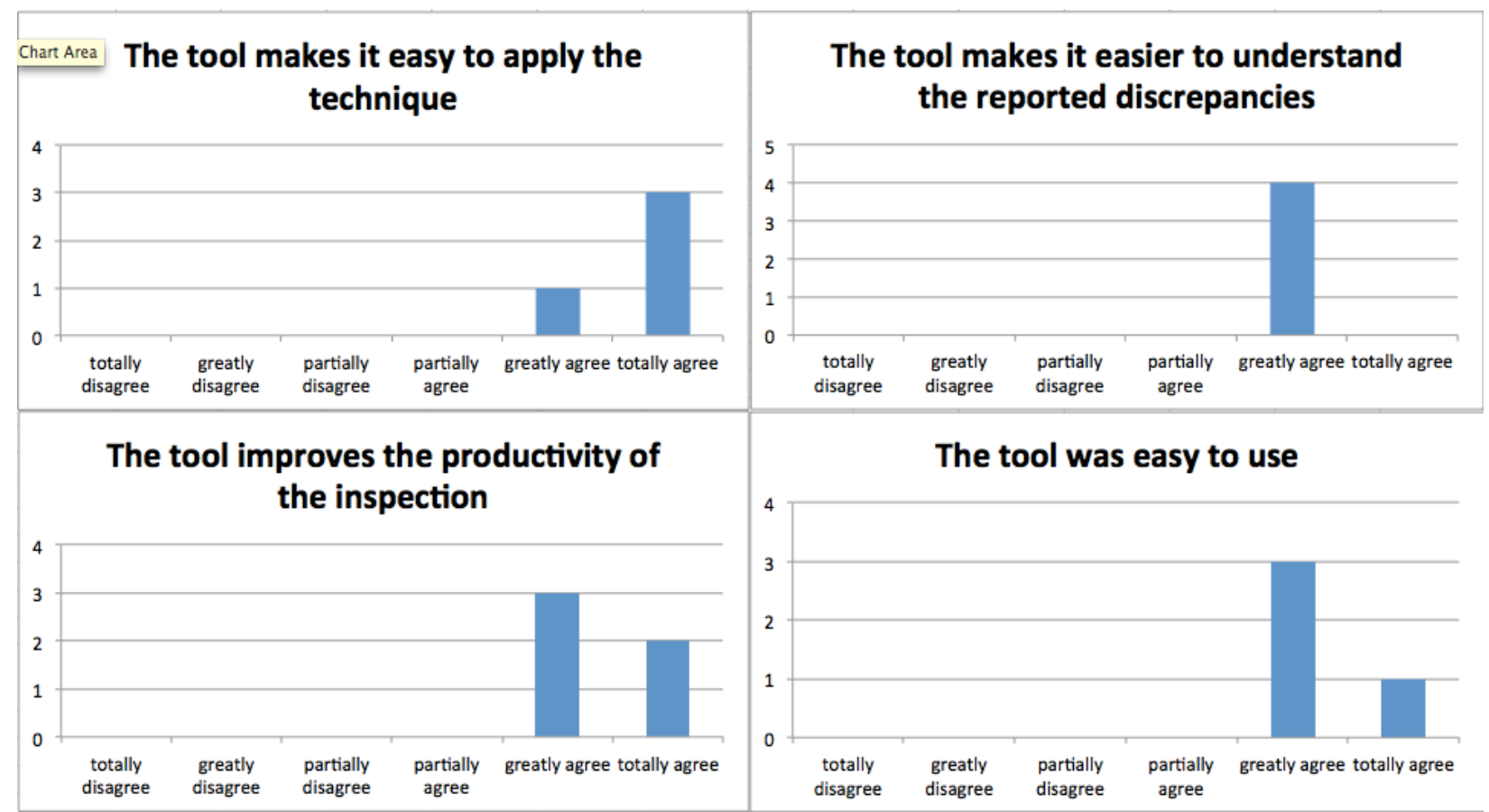

Figure 11: Qualitative results from the second empirical study

The results show that all inspectors greatly or totally agreed with the statements regarding the usefulness and ease of use of the tool. Furthermore, all inspectors stated that they would use the tool again, which corroborates the results from the first empirical study.

One of the limitations from this study is the number of subjects. As the number was low we were not able to perform a statistical method and could not generalize its results either. However, they are positive indicators of the feasibility of the WDP Tool to support the inspection of Web applications in an industrial environment.

\section{Comparison with Related Tools}

In [22], Santos and Conte presented the APIU (Apoio ao Processo de Inspeção de Usabilidade, or Support to the Usability Inspection Process) tool that supports the usability inspection process. The APIU, differently from the WDP Tool, aims to support the overall inspection process, from the planning to the prioritization and correction of the encountered defects.

As the main advantage of using the WDP Tool over the APIU tool, we can say that, as the WDP Tool is integrated to the browser, it allows an easier interaction, enabling inspectors to report the discrepancies without leaving the application context they are inspecting. Furthermore, despite the fact that the APIU allows the inspector to upload an image to show the identified discrepancy, it is not able to automatically take a screenshot as in the WDP Tool.

In [9], Fernandez et al. proposed the Interactive Web Evaluation (Interactive WE) tool. The Interactive WE supports the inspection process of the Web Evaluation Question Technique (WE-QT), a derivation of the WDP technique in the shape of questions to assist novice inspectors. As the WDP Tool, the Interactive WE supports the defect detection phase, albeit with the use of another technique.

The main advantage of using the WDP Tool over the Interactive WE is its integration with the Web browser. Furthermore, despite being able to capture the system screen, this screen shot always grabs the whole screen and not the inspected application. Furthermore, the Interactive WE does not allow inspectors to edit the screen shot to point the identified defect, which makes the information less specific. Finally, the report generated by the Interactive WE is in text format and does not provide links to the screen shots, which is a feature that is supported by the WDP tool.

\section{Conclusion}

This paper presented the empirical evaluation of a tool to support usability inspections, the WDP Tool. This tool aims to solve the issues regarding the application of the WDP technique. The results from the observational studies 
offer a positive indication on the WDP tool's feasibility when used in an industrial environment. We hope such indications can encourage its use by practitioners in the software development industry.

The main contribution of this work is to identify and solve the main issues in the WDP technique application by offering the WDP tool. Furthermore, the WDP tool can also be used integrated with other tools for software inspections (such as ISPIS) or the WDP technique (WDP content consult assistant), extending their functionalities and increasing their potential use.

As future work we intend to increase the possibilities of using the WDP Tool by: (1) developing tool versions for different browsers, and (2) doing more empirical studies to compare the inspection efficiency among inspection teams using and not using the WDP tool. In these studies, it will be also important to compare not only the detection phase efficiency, but also the discrimination and defect correction phases. The reason for these comparisons is the screenshot capability, which can facilitate the understanding of discrepancies and reduce the effort needed in other inspection stages.

\section{Acknowledgements}

The authors would like to dedicate this paper to Professor Alberto Luiz Coimbra, in the $50^{\text {th }}$ anniversary of COPPE (1963-2013), the Graduate School of Engineering of the Federal University of Rio de Janeiro. Furthermore, we would like to thank Miguel Lannes Fernandes for the opportunity he gave us, allowing us to carry out the in vivo study in his firm, as well as CNPq, FAPEAM, and the COPPETEC Foundation/SIGIC Project for their valuable support in the development of the WDP tool.

\section{References}

[1] V. Basili, and D. Rombach, "The tame project: towards improvement-oriented software environments," IEEE Transactions on Software Engineering, vol. 14, no. 6, pp. 758 - 773,1988.

[2] T. Conte, J. Massolar, E. Mendes, G. H. Travassos, "Web Usability Inspection Technique Based on Design Perspectives", IET Software Journal, v. 3, n. 2, pp. 106-123, 2009.

[3] T. Conte, V. Vaz, J. Massolar, E. Mendes, and G. Travassos, "Improving a Web Usability Inspection Technique using Qualitative and Quantitative Data from an Observational Study," in Proc. XXIII Brazilian Symposium on Software Engineering - SBES, Fortaleza - Brazil, 2009, pp. 227-235.

[4] T. Conte, V. Vaz, D. Zanetti, G. Santos, A. Rocha, and G. Travassos, "Applying the Technology Acceptance Model to a Usability Inspection Technique," in: Proc. IX Brazilian Symposium on Software Quality -SBQS, Belém, 2010, pp. 367-374. (in Portuguese)

[5] F.Davis, "Perceived usefulness, perceived ease of use, and user acceptance of Information Technology,"MIS Quarterly, vol. 13, no. 3, pp. 319-339, 1989.

[6] A. Dix, J. Finlay, G. Abowd, R. Beale, "Human-Computer Interaction", Ed. Pearson/ Prentice Hall, 3 rd Ed., 2004.

[7] J. de la Vara, K. Wnuk, R. Berntsson-Svensson, J. Sánchez, and B. Regnell, "An Empirical Study on the Importance of Quality Requirements in Industry,"in: XXIII International Conference on Software Engineering and Knowledge Engineering - SEKE 2011, Miami Beach, USA, 2011, pp. 438 - 443.

[8] A. Fernandez, E. Insfran, S. Abrahao, "Usability evaluation methods for the Web: A systematic mapping study. Information and Software Technology”, Volume 53, Issue 8, 2011.

[9] P. Fernandes, L. Rivero, B. Bonifacio, D. Santos and T. Conte, "Evaluating a new Approach for Usability Inspection through a Qualitative and Quantitative Analysis," in Proc.VIII Experimental Software Engineering Latin American Workshop, Rio de Janeiro, 2011. (in Portuguese)

[10] J. Francese, "Development of a Tool Support for the Usability Defects Detection in Web Systems," in Proc. Jornada Giulio Massarani de Iniciação Científica, Artística e Cultural - JIC, Rio de Janeiro - Brazil, 2008.(in Portuguese)

[11] International Organization for Standardization - ISO/IEC 9126-1, "Information Technology - Software Product Quality. Part 1: Quality Model,” 1999.

[12] M. Kalinowski, and G. Travassos, “A Computational Framework for Supporting Software Inspections," in Proc. XIX IEEE Conference on Automated Software Engineering, Linz - Austria, 2004, pp. 46-55. 
[13] O. Laitenberger, and H. Dreyer, "Evaluating the Usefulness and the Ease of Use of a Web-based Inspection Data Collection Tool,” in: Proc. X Software Metrics Symposium, Bethesda - MD, 1998, pp. 122-132.

[14] D. Lavery, G. Cockton, and M. Atkinson, "Comparison of evaluation methods using structured usability problem reports," Behaviour \& Information Technology, vol. 16, no. 4, pp. 246 - 266, 1997.

[15] Mozilla Developer Center - Extensions. Available at: https://developer.mozilla.org/enUS/docs/Extensions(last access 15/02/2013).

[16] Mozilla Public License Version 1.1. Available at:http://www.mozilla.org/MPL/1.1/ (last access 16/03/2012).

[17] MPS.Br - Brazilian Software Process Improvement. Available at:www.softex.br/mpsbr/ (last access $16 / 03 / 2012)$.

[18] J. Nielsen, "Heuristic evaluation," J. Nielsen, and R. Mack, (Eds.), John Wiley \& Sons, 1994, Usability Inspection Methods, 1994.

[19] L. Olsina, G. Covella, and G. Rossi, "Web Quality,"E. Mendes, andN. Mosley (Eds), Web Engineering, Springer Verlag, 2006.

[20] Paint - Microsoft Windows. Available at: http://windows.microsoft.com/enUS/windows7/products/features/paint (last access 15/02/2013).

[21] P. Polson, C. Lewis, J. Rieman, C. Wharton, "Cognitive Walkthroughs: a method for theory-based evaluation of users interfaces”, in International Journal of Man-Machine Studies, 1992.

[22] F. Santos, and T. Conte, "Evolving na Assistant for Usability Inspection through Empirical Studies," in Proc. XIV Iberian-American Congress in Software Engineering - CibSE, Rio de Janeiro, 2011, pp 23. (in Portuguese)

[23] C. Sauer, D. Jeffery, L. Land, and P. Yetton, "The Effectiveness of Software Development Technical Review: A Behaviorally Motivated Program of Research,"IEEE TSE, vol. 26, no. 1, pp. 1-14, 2000.

[24] F. Shull, J. Carver, and G. Travassos, “An empirical methodology for introducing software processes,” $A C M$ SIGSOFT Software Engineering Notes, vol. 26, no. 5, pp. 288-296, 2001.

[25] V. Vaz, T. Conte, A. Bott, E. Mendes, and G. Travassos, "Usability Inspection in Software Development Organizations - A Practical Experience," in Proc. Brasylian Simpoium in Software Quality -SBQS, Florianopolis, 2008, pp. 369-378. (in Portuguese)

[26] V.Venkatesh, M. Morris, and D. Davis, "User acceptance of information technology: Towards an unified view,"MIS Quarterly, vol. 27, no. 3, pp. 425-478, 2003.

[27] Z. Zhang, V. Basili, and B. Shneiderman, "Perspective-based Usability Inspection: An Empirical Validation of Efficacy,”Empirical Software Engineering: An International Journal, vol. 4, no. 1, pp. 43-70, 1999.

[28] WDPTool: Add-ons for Firefox. Available at: https://addons.mozilla.org/en-us/firefox/addon/wdp-tool/ (last access 15/02/2013).

[29] W3Counter - Global Web Stats.Available at:http://w3counter.com/globalstats.php (last access 12/03/2012). 
